SHORT REPORT

\title{
Pseudomyxoma peritonei: unusual origin from an ovarian mature cystic teratoma
}

\author{
N Pranesh, L P Menasce, M S Wilson, S T O'Dwyer
}

J Clin Pathol 2005;58:1115-1117. doi: 10.1136/jcp.2004.025148

Pseudomyxoma peritonei (PMP) is classified into pathologically and prognostically distinct categories, such as disseminated peritoneal adenomucinosis (DPAM) and peritoneal mucinous carcinomatosis. There is overwhelming evidence that DPAM arises from a mucinous adenoma of the appendix. The one exception to this is the presentation of a mature ovarian cystic teratoma as PMP where the appendix is normal. This report describes such a case and discusses the presentation, histopathology, and treatment options.

$\mathrm{P}$ seudomyxoma peritonei (PMP) is classified into pathologically and prognostically distinct categories, such as disseminated peritoneal adenomucinosis (DPAM) and peritoneal mucinous carcinomatosis. There is overwhelming evidence that DPAM arises from a mucinous adenoma of the appendix. The one exception to this is the presentation of a mature ovarian cystic teratoma as PMP where the appendix is normal. We report such a case and discuss the presentation, histopathology, and treatment options.

\section{CASE REPORT}

A 39 year old woman presented with a three month history of abdominal distension and an episode of abnormal per vaginal bleeding. Clinical examination revealed a mass arising out of the pelvis and ultrasound examination demonstrated a large multicystic complex mass containing fluid and solid components, arising from the left ovary. The tumour marker CAl25 was normal at $11 \mathrm{KU} /$ litre. At laparotomy, copious amounts of free mucin and tenacious mucinous deposits were seen on the surface of the omentum and bowel. There was a $10 \times 12 \mathrm{~cm}$ left ovarian mass and a small right ovarian cyst. Peritoneal lavage, hysterectomy, bilateral salpingo-oophorectomy, omentectomy, and appendicectomy were performed. After an uneventful recovery she was referred to our PMP centre for further management. Nine months after surgery, an abdominal computed tomography scan shows no evidence of PMP and tumour markers such as carcinoembryonic antigen, CA125, and CA19-9 are within normal limits.

\section{HISTOPATHOLOGY}

Macroscopic examination revealed a multiloculated, cystic, left ovarian mass measuring $14 \times 12.5 \times 7.5 \mathrm{~cm}$. The cut surfaces revealed multiple cysts with smooth walls containing mucinous material. One of the cysts contained sebum and hairs. The appendix measured $4 \mathrm{~cm}$ with no focal lesions identified on the cut surface.

Paraffin wax embedded sections of the left ovary confirmed the presence of a mature cystic teratoma (dermoid cyst) and a mucinous tumour. The teratomatous component consisted of a cyst lined by keratinising squamous epithelium containing skin appendages, such as sebaceous and apocrine glands (fig lA). The mucinous component was
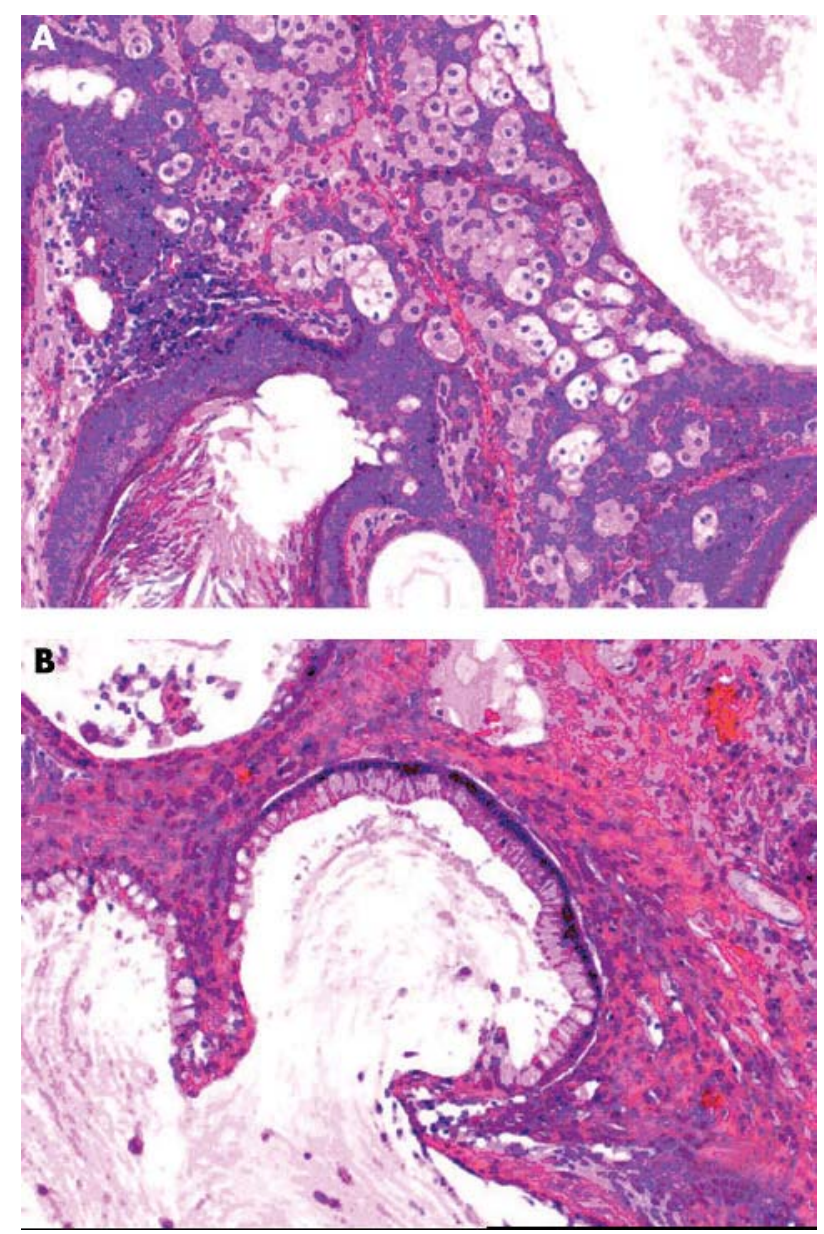

Figure 1 (A) Teratomatous component; (B) mucinous component.

multiloculated with the cysts lined by a single layer of mucin secreting epithelium (fig 1B). There were intestinal changes characterised by the presence of goblet cells and occasional Paneth cells. Sections of the appendix demonstrated mucinous material on the serosal surface. No abnormal epithelial proliferation was seen along the entire length of its lumen. The surfaces of the uterus and omentum also demonstrated mucinous material but without epithelial elements.

On immunoperoxidase staining, the mucinous epithelium in the ovary was cytokeratin 20 (CK20), carcinoembryonic antigen, and BerEP4 positive and CK7 negative.

Abbreviations: CK, cytokeratin; DPAM, disseminated peritoneal adenomucinosis; PMCA, peritoneal mucinous carcinomatosis; PMP pseudomyxoma peritonei 


\section{DISCUSSION}

Although the association of appendiceal mucinous tumours/ mucocoele with PMP has long been recognised, ${ }^{12}$ the true origin of PMP in women who have simultaneous appendiceal and ovarian tumours has been a dilemma. Advances in immunohistochemical and molecular genetic techniques have provided compelling evidence that the source of PMP is an appendiceal mucinous tumour. ${ }^{3-6}$ In the past decade, detailed classifications of PMP have emerged with descriptions of distinct pathological characteristics of prognostic relevance. PMP has been classified into DPAM, characterised histologically by bland to low grade adenomatous mucinous epithelium and an associated appendiceal mucinous neoplasm; peritoneal mucinous carcinomatosis (PMCA), often with an associated mucinous adenocarcinoma of the gastrointestinal tract; and PMCA with intermediate or discordant features, which has combined features of DPAM and PMCA. It has been suggested that the term PMP should be used to describe a clinical syndrome of mucinous ascites along with the pathological diagnosis of DPAM. ${ }^{8}$

There are four important points to consider in the above case. (1) The appendix was macroscopically and microscopically normal, suggesting a primary ovarian mucinous tumour. (2) The morphology and immunophenotype of the epithelium lining the mucinous cysts in the ovary suggested a gastrointestinal origin (CK20+/CK7-). (3) The presence of squamous epithelium and skin appendages in the ovary indicated the presence of a mature cystic teratoma. (4) Peritoneal mucinous deposits were devoid of lining mucinous epithelium.

The association of ovarian teratomas, mucinous ovarian tumours, and PMP has been reported in two recent studies. The first was a study of mucinous tumours of the ovary (of intestinal type) in which three cases of PMP with an associated ovarian dermoid cyst were reported. ${ }^{9}$ Of these, two had a normal appendix, did not demonstrate peritoneal DPAM lesions, and had no evidence of disease recurrence after five years and 16 years. The third patient in whom neither the appendix was removed nor the peritoneal lesions sampled was alive without clinical evidence of recurrence after a follow up period of four years and nine months. The CK immunophenotype of these tumours was not reported.

The second study reported three cases of mucinous tumours in ovarian cystic teratomas, ${ }^{10}$ where the appendix was microscopically normal and the mucinous tumours had a CK20+/CK7- immunophenotype. The peritoneal lesions had histological features suggestive of PMP/DPAM in two cases and PMCA with intermediate or discordant features in the third. No follow up data were reported for this group of patients.

\section{"The lack of lining epithelium in the peritoneal mucinous deposits, combined with normal tumour markers and normal imaging, make it difficult to outline the treatment that should be offered to our patient"}

An appendiceal mucinous neoplasm can be found in almost all cases of DPAM/PMP with the notable exception of those arising in the gastrointestinal component of a mature cystic teratoma. Primary ovarian mucinous tumours are usually CK7 positive and show variable expression of CK20, ${ }^{11}$ unlike those associated with mature teratomas, which have the CK20+/CK7- phenotype. ${ }^{10}$

The above factors, particularly the lack of lining epithelium in the peritoneal mucinous deposits, combined with normal tumour markers and normal imaging, make it difficult to outline the treatment that should be offered to our patient. If her diagnosis is PMP/DPAM, then she is at risk of recurrent
Take home messages

- We report a patient with a mature ovarian cystic teratoma in whom the appendix was normal and who presented with pseudomyxoma peritonei

- The pathological, histological, and tumour marker characteristics of our patient make it difficult to classify this tumour into a distinct category (such as disseminated peritoneal adenomucinosis or peritoneal mucinous carcinomatosis) and advise the patient on the appropriate treatment

- Therefore, we have decided to take a "wait and watch" approach, and plan to carry out six monthly tumour marker measurements and annual computed tomography scans

PMP and should undergo cytoreduction and hyperthermic intraperitoneal chemotherapy, the currently accepted treatment. ${ }^{12}$ However, the similarity of her pathology to that of the three patients in the first of the above studies ${ }^{9}$ suggests that she could be treated conservatively, because up to the time of reporting PMP had not recurred in these patients.

The above considerations have led to a "wait and watch" approach to the management of our patient, with plans to carry out six monthly tumour marker measurements and annual computed tomography scans.

\section{Authors' affiliations}

N Pranesh, M S Wilson, S T O'Dwyer, Department of Surgery, Christie Hospital NHS Trust, Withington, Manchester M20 4BX, UK

L P Menasce, Department of Pathology, Christie Hospital NHS Trust

Full consent was given for the publication of this report.

Correspondence to: Mr N Pranesh, PMP Service, Surgical Theatres, Christie Hospital, Wilmslow Road, Withington, Manchester M20 4BX, UK; Nagarajan.Pranesh@christie-tr.nwest.nhs.uk

Accepted for publication 22 December 2004

\section{REFERENCES}

1 Long RT, Spratt JS Jr, Dowling E. Pseudomyxoma peritonei. New concepts in management with a report of seventeen patients. Am J Surg 1969;117:162-9.

2 Gibbs NM. Mucinous cystadenoma and cystadenocarcinoma of the vermiform appendix with particular reference to mucocele and pseudomyxoma peritonei. $J$ Clin Pathol 1973;26:413-21.

3 O'Connell JT, Tomlinson JS, Roberts AA, et al. Pseudomyxoma peritonei is a disease of MUC2-expressing goblet cells. Am J Pathol 2002;161:551-64.

4 Prayson RA, Hart WR, Petras RE. Pseudomyxoma peritonei. A clinicopathologic study of 19 cases with emphasis on site of origin and nature of associated ovarian tumors. Am J Surg Pathol 1994;18:591-603.

5 Ronnett BM, Shmookler BM, Diener-West M, et al. Immunohistochemical evidence supporting the appendiceal origin of pseudomyxoma peritonei in women. Int J Gynecol Pathol 1997;16:1-9.

6 Szych C, Staebler A, Connolly DC, et al. Molecular genetic evidence supporting the clonality and appendiceal origin of Pseudomyxoma peritonei in women. Am J Pathol 1999; 154:1849-55.

7 Ronnett BM, Zahn CM, Kurman RJ, et al. Disseminated peritoneal adenomucinosis and peritoneal mucinous carcinomatosis. A clinicopathologic analysis of 109 cases with emphasis on distinguishing pathologic features, site of origin, prognosis, and relationship to "pseudomyxoma peritonei". Am J Surg Pathol 1995; 19:1390-408.

8 Ronnett BM, Yan H, Kurman RJ, et al. Patients with pseudomyxoma peritonei associated with disseminated peritoneal adenomucinosis have a significantly more favorable prognosis than patients with peritoneal mucinous carcinomatosis. Cancer 2001;92:85-91.

9 Lee KR, Scully RE. Mucinous tumors of the ovary: a clinicopathologic study of 196 borderline tumors (of intestinal type) and carcinomas, including an evaluation of 11 cases with "pseudomyxoma peritonei". Am J Surg Pathol 2000;24:1447-64. 
10 Ronnett BM, Seidman JD. Mucinous tumors arising in ovarian mature cystic teratomas: relationship to the clinical syndrome of pseudomyxoma peritonei. Am J Surg Pathol 2003;27:650-7.

11 Ji H, Isacson C, Seidman JD, et al. Cytokeratins 7 and 20, Dpc4, and MUC5AC in the distinction of metastatic mucinous carcinomas in the ovary from primary ovarian mucinous tumors: Dpc4 assists in identifying metastatic pancreatic carcinomas. Int J Gynecol Pathol 2002;21:391-400.

12 Sugarbaker PH. Cytoreductive surgery and perioperative intraperitoneal chemotherapy as a curative approach to pseudomyxoma peritonei syndrome. Tumori 2001;87:S3-5. 